\section{Viral checkmate}

Researchers use the tremendous combinatorial diversity of recombinant antibody libraries to enable mapping of viral trajectories in vitro.

In the cat-and-mouse game between a virus and the host immune system, the latter is necessarily always reactive. It cannot anticipate the routes that the virus might take and prepare its response accordingly. But with a little help from modern molecular biology, it may be possible to do so in vitro. This is what a collaborative group at The Scripps Institute, including Richard Lerner, Tobin Dickerson, Kim Janda and Sydney Brenner, have set out to do. They have named the approach 'checkmate analysis'.

The essence of the idea is simple. "Neutralization games are played out in the loops of viral proteins," explains Lerner, "and the mutation space that a virus can cover is very large. Infection studies in tissue culture aren't able to saturate that space. But with combinatorial antibody libraries and a replication system in phage, we thought we could map out the viral trajectory."

The researchers initially focused on the trimeric hemagglutinin (HA) protein of the influenza virus. Using a suppressible amber codon in the linker between HA and the phage coat protein pIII, they could achieve simultaneous expression of both soluble HA monomers and the monomer fused to the phage coat, resulting in functional HA trimer on the phage surface. Having achieved this, the authors propose that it will be simple, using standard phage display and mutagenesis, to screen for HA variants that 'escape' the effect of neutralizing antibodies (Fig. 1). The antibody library can in turn be screened for variants that capture the escaping viral antigens. These are added to the next selection cycle, thus increasing the challenge to the virus, which is screened again for a further escape response. Thus, both the possible viral and antibody trajectories can be mapped in vitro.

"An important consequence is that this looks both forward and backward in time," says Dickerson, first author on the paper proposing this approach. "You will identify all possible routes the virus

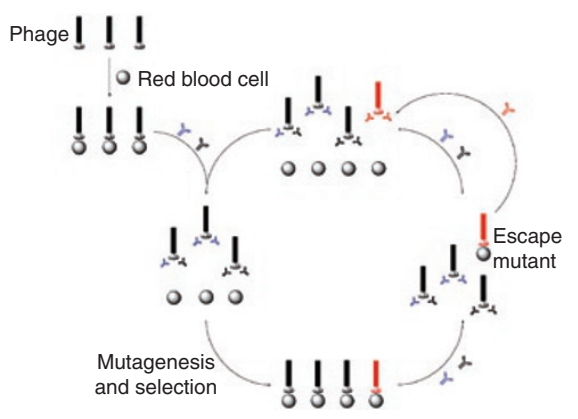

Figure 1 | Immunological checkmate analysis. Iterative cycles of mutagenesis and panning to map trajectories of phage-displayed HA escape variants in vitro. Reprinted with permission from the National Academy of Sciences, USA. Copyright 2007.

can take, so you can see both where it has been, and where it might go in the future." Clearly, identification by checkmate analysis of HA variations known to have been acquired in the field will be a key step in validating the method. If, as the researchers hope, the method allows predictive mapping of viral paths, this opens up the exciting possibility that therapeutic options may become available ahead of time for viruses evolving in the field.

But this is not all that checkmate analysis could be useful for. "It occurred to us," says Lerner, "that this could be a general screening method for any proteinprotein interaction." Using an ingeniously simple selection system, in which bacterial growth is dependent on disrupting the interaction between a displayed receptor and its ligand, the researchers are now using checkmate analysis to screen smallmolecule libraries for agents that interfere with several receptor-ligand interactions. The beauty of the approach, says Lerner, is that "you bring single-molecule screening into the hands of anybody who wants it. It will really find its place in the academic arena, because you don't need expensive equipment to do this. All you need is phage and some toothpicks."

\section{Natalie de Souza}

\section{RESEARCH PAPERS}

Dickerson, T.J. et al. Phage escape libraries for checkmate analysis. Proc. Natl. Acad. Sci. USA 104, 12703-12708 (2007). 